\title{
Differential cross-complementation patterns of Escherichia coli and Neisseria gonorrhoeae RecA proteins
}

\author{
Elizabeth A. Stohl, Leslie Blount and H. Steven Seifert
}

Department of

Microbiology-Immunology,

The Feinberg School of Medicine, Northwestern University, 303 E Chicago Avenue, Chicago, IL 60611, USA Author for correspondence: H. Steven Seifert. Tel: +1 3125039788. Fax: +1 3125031339.
e-mail: h-seifert@northwestern.edu

Keywords: DNA repair, pilus antigenic variation, homologous recombination, recX

\section{INTRODUCTION}

Homologous recombination is an essential function in all organisms. It is central to both sexual and asexual genetic exchange, maintenance of chromosomal integrity, DNA repair and DNA replication. In Escherichia coli, RecA has a pivotal role in homologous recombination mediated by either the RecBCD or the RecF pathway. RecA is also integral to the repair of damaged DNA and coordinates error-prone DNA repair via SOS mutagenesis through interactions with several other proteins (Cox, 1993; Kowalczykowski et al., 1994). The generation of a battery of mutant E. coli recAs in conjunction with the elucidation of the E. coli RecA crystal structure (Story et al., 1992) has allowed the assignment of functional domains and residues to the RecA protein. Further understanding of RecA protein structure and function has been enhanced by both functional complementation assays and sequence comparisons of recA homologues from other organisms (Miller \& Kokjohn, 1990).

Abbreviation: Gc, Neisseria gonorrhoeae.
The obligate pathogen Neisseria gonorrhoeae (Gc) expresses type IV pili, a virulence factor that mediates the initial attachment of the bacterium to the host epithelium. Pili are essential for full infectivity (Kellogg et al., 1963; Swanson et al., 1987) and are required for full DNA transformation competence (Seifert et al., 1990; Sparling, 1966). The Gc pilus is mainly composed of the pilE gene product, pilin (Swanson et al., 1971), and antigenic variation of the pilus occurs when the sequence of pilE is altered, leading to changes in the amino acid sequence of the pilin protein (Virji \& Heckels, 1983). Antigenic variation of the pilus can also result in changes in pilus-dependent colony morphology (Long et al., 1998). Therefore, changes in gonococccal colony morphology can be used to estimate the frequency of pilin antigenic variation (Haas \& Meyer, 1986; Swanson et al., 1986).

Pilus antigenic variation is a RecA-dependent process (Koomey \& Falkow, 1987; Koomey et al., 1987). The genome of Gc contains multiple, incomplete, silent copies of the pilin gene $(p i l S)$, portions of which are recombined unidirectionally into the expressed pilE gene (Haas \& Meyer, 1986; Hagblom et al., 1985). 
Molecular models for these unique recombination reactions propose a pilE/pils hybrid intermediate, which has a crossover at a small region of conserved sequence (Howell-Adams et al., 1996) and targets recombination with the recipient pilE gene (HowellAdams \& Seifert, 2000). The recA gene of Gc was identified by its ability to partially complement an E. coli recA mutant deficient in both homologous recombination and DNA repair functions (Fyfe \& Davies, 1990; Koomey \& Falkow, 1987). The Gc RecA protein is $65 \%$ identical and $81 \%$ similar to the E. coli RecA protein and shows significant sequence similarity to other RecA proteins. Sequence comparisons of RecA proteins from many bacteria have revealed the carboxy terminus of RecA to be the most divergent portion of the protein (Miller \& Kokjohn, 1990), and it is this portion of RecA that is the most variable between the Gc and E. coli RecA proteins (Fyfe \& Davies, 1990).

In $\mathrm{Gc}$, the RecA protein is essential for DNA transformation, DNA repair and pilus antigenic variation (Koomey \& Falkow, 1987; Koomey et al., 1987). In Gc, genetic transfer of chromosomal markers is achieved through DNA transformation, whereas in E. coli transfer of chromosomal genes is accomplished through transduction or conjugation. However, in both bacteria these processes are dependent on the RecBCD pathway of homologous recombination (Kowalczykowski et al., 1994; Mehr \& Seifert, 1998), and DNA transformation in Gc is also affected by mutation of recN (Skaar et al., 2002). In Gc, efficient DNA repair requires genes of the RecBCD pathway $(r e c B$, rec C, $r e c D)$ and the RecF-like pathway (recJ, recN, recO and recQ) (Hill, 2000; Mehr \& Seifert, 1998; Skaar et al., 2002). In contrast, mutations in E. coli recD or recQ do not affect DNA repair (Kowalczykowski et al., 1994). Additionally, the SOS system of DNA repair appears to be absent in Gc (Black et al., 1998). Thus, the mechanisms utilized for horizontal chromosomal DNA transfer in Gc and E. coli appear to be similar, but DNA repair mechanisms diverge more between these two bacteria.

Pilus antigenic variation is a Gc-specific, recA-mediated cellular process. In addition to the RecA protein, the RecF-like pathway is essential for pilus antigenic variation (Mehr \& Seifert, 1998; Skaar et al., 2002; Hill \& Grant, 2002), and conflicting reports suggest either that the $\mathrm{RecBCD}$ pathway is not required for efficient antigenic variation (Mehr \& Seifert, 1998; Hill \& Grant, 2002), or that it plays an inhibitory role in mediating colony phenotype switches (Chaussee et al., 1999). Finally, the $\mathrm{RdgC}$ protein is involved in pilus antigenic variation and cell morphology (Mehr et al., 2000).

A gene designated $r e c X$ has been identified downstream of recA in many organisms, and in some instances the coding regions for the recA and recX genes overlap (De Mot et al., 1994; Yang et al., 2001). In mycobacteria and Streptomyces lividans the genes are cotranscribed (Papavinasasundaram et al., 1997; Vierling et al., 2000). Further work has led to the hypothesis that RecX acts by downregulating RecA activity (Sano, 1993;
Papavinasasundaram et al., 1998; Vierling et al., 2000), though this hypothesis has not been directly tested, and a recX mutation in $S$. lividans does not affect any RecA-mediated processes or RecA expression levels. E. coli contains a RecX homologue located downstream of recA, designated oraA (Zaitsev et al., 1994). Due to the established nomenclature of other RecX homologues, we will refer to the E. coli ora $A$ as recX in this paper. No work has to our knowledge been published detailing the role of the E. coli recX in DNA recombination and repair. However, in Gc the recX gene is located in a different chromosomal locus than recA, and a recX mutant exhibits decreases in the RecA-mediated phenotypes of DNA repair, DNA transformation and pilus antigenic variation (Stohl \& Seifert, 2001).

If the different cellular processes catalysed by RecA require specific interactions with other proteins or involve unique biochemical properties of the RecA proteins themselves, then expression of RecA from a different species may not result in full complementation. Furthermore, since DNA repair may occur through different mechanisms in Gc and E. coli and since pilus antigenic variation is a process unique to $\mathrm{Gc}$, it would be interesting to determine whether expression of E. coli recA in Gc could mediate each of these cellular processes. To test these hypotheses we expressed the E. coli and Gc recA genes in both organisms under the control of the lac regulatory system. In each organism both RecA proteins were able to act as recombinases, but they differed markedly in their ability to mediate DNA repair. Studies of three strains of Gc expressing the E. coli recA alone, an E. coli recA genomic clone that additionally contains the E. coli recX gene, or E. coli recA and overexpressed E. coli recX on separate transcripts revealed differential RecA phenotypes that were reflected by varying RecA protein levels.

\section{METHODS}

Bacterial strains and growth conditions. E. coli strain $\mathrm{DH} 5 \alpha$ (Gibco-BRL), used to propagate plasmids, and all other E. coli strains, were grown on Luria-Bertani (LB) broth or agar at $37^{\circ} \mathrm{C}$ unless otherwise indicated. Minimal medium (M9) agar was prepared according to Miller (1992). E. coli strains LB1 and LB2, $\Delta r e c A$ derivatives of AB1157 (DeWitt \& Adelberg, 1962), and DM49 (Mount et al., 1972), were constructed by transducing AB1157 and DM49 to Tet ${ }^{\mathrm{R}}$ with P1 phage grown on JC10289 (Csonka \& Clark, 1979) to introduce the $\Delta($ rec $A-s r l) 306:$ : Tn10 marker. Gc strains were grown on Gc Medium Base (Difco) plus Kellogg supplements (GCB) $(22.2 \mathrm{mM}$ glucose, $0.68 \mathrm{mM}$ glutamine, $0.45 \mathrm{mM}$ cocarboxylase, $1.23 \mathrm{mM} \mathrm{Fe}\left(\mathrm{NO}_{3}\right)_{3}$; all from Sigma) (Kellogg et al., 1963) at $37^{\circ} \mathrm{C}$ in $5 \% \mathrm{CO}_{2}$, or in Gc Liquid (GCBL) medium $[1.5 \%$ protease peptone no. 3 (Difco), $0.4 \%$ $\mathrm{K}_{2} \mathrm{HPO}_{4}$ (Fisher), $0 \cdot 1 \% \mathrm{KH}_{2} \mathrm{PO}_{4}$ (Fisher), $0 \cdot 1 \% \mathrm{NaCl}$ (Fisher)] with Kellogg Supplements and 0.042\% sodium bicarbonate (Sigma) at $37^{\circ} \mathrm{C}$ with rotation. All gonococcal strains used in this study were derivatives of strain FA1090 1-81-S2 with matched pilE sequences (Seifert et al., 1994) determined as described by Stohl \& Seifert (2001). Antibiotics were added at the following concentrations. For E. coli: chloramphenicol, 

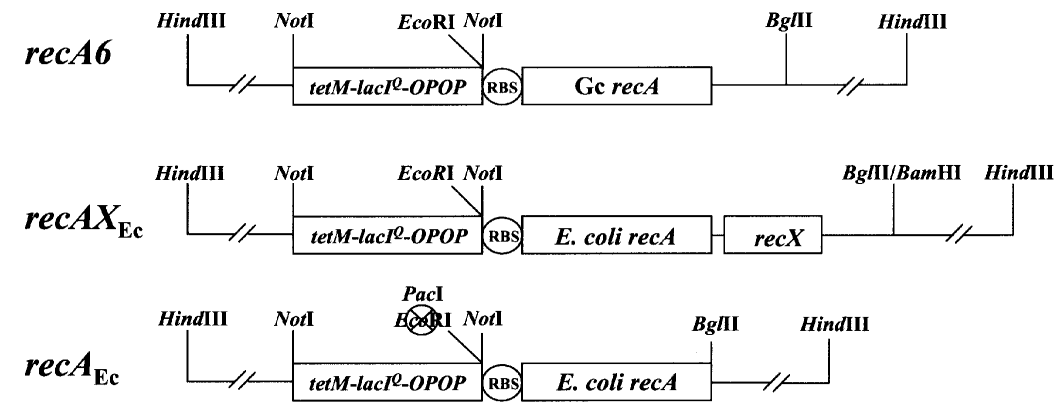

Cg8 locus $L_{\text {ermC-lacI }}^{\text {ClaI }}$
Fig. 1. Maps of $\operatorname{rec} A$ alleles. $l a c l^{\mathrm{Q}}$ encodes the Lac repressor, OPOP represents the dual tac and UV5 operator and promoter sequences, and RBS indicates the ribosomebinding site. Replacement of the EcoRI restriction site with a Pacl site, and creation of a new site by $B g / l 1 / B a m H I$ ligation, is indicated (see Methods). The recA6 allele was previously described (Seifert, 1997). In Gc, constructs are carried in either the Gc recA or the Cg8 locus, as indicated. In E. coli, alleles $\operatorname{rec} A X_{\mathrm{EC}}$ and $\operatorname{rec} A 6$ are carried on the pACYC184 derivative pVD300 (Koomey \& Falkow, 1987).
$30 \mathrm{mg} \mathrm{l}^{-1}$; erythromycin (Erm), $275 \mathrm{mg} \mathrm{l}^{-1}$; kanamycin, $40 \mathrm{mg} \mathrm{l}^{-1}$. For Gc: Erm, $2 \mathrm{mg} \mathrm{l}^{-1}$; spectinomycin (Spc), $50 \mathrm{mg} \mathrm{l}^{-1}$; nalidixic acid (Nal), $1 \cdot 25 \mathrm{mg} \mathrm{l}^{-1}$. IPTG (Diagnostic Chemicals) was supplied at either $1.5 \mathrm{mM}$ (for E. coli) or $1 \mathrm{mM}$ (for Gc) in solid agar or liquid medium.

DNA manipulations and analysis. Standard procedures were performed as described by Sambrook et al. (1989). Plasmid DNA was isolated from strains of E. coli using Qiagen plasmid kits. DNA was isolated from agarose gels using Qiaquick gel extraction kits (Qiagen). Enzymes were used according to the manufacturers' directions (Promega and New England Biolabs). E. coli strains were transformed by electroporation using the Gene Pulser II Electroporation System (BioRad) according to the manufacturer's specifications. For Southern blot analysis, DNA was transferred to Magnagraph nylon membrane according to the manufacturer's specifications (Micro Separations). Labelling of digoxigeninlabelled dUTP probes, hybridization, and chemiluminescent blot development were performed using the Genius kit (Roche Pharmaceuticals) according to the manufacturer's instructions. Sequencing reactions were performed using the Big-Dye Terminator Cycle Sequencing kit (Perkin-Elmer) and sequencing products were separated on an ABI model 377 automated DNA sequencer. DNA sequences were aligned and compiled using Lasergene software (DNASTAR) and VectorNTI software (Informax).

Construction of plasmids pVD300recAX $X_{\mathrm{Ec}}$ and pEAS3/rec $A_{\mathrm{Ec}}$ and expression from the Gc recA locus. The $E$. coli $r e c A / r e c X$ genomic region was amplified from plasmid pERA (R. D. Porter, unpublished) by PCR using primers M13REV $\left(5^{\prime}-\right.$ CAGGAAACAGCTATGACCATGATTAC-3') and ECRECANOTFOR (5'-GGCGGCCGCAGGAGTAAAAATGGCTATC-3'), which introduces a Not I site (underlined), digested with BamHI and NotI, and cloned between the NotI and BglII sites of pVD300NL1 (Seifert, 1997) to replace the Gc recA gene. The insert was sequenced to verify that no errors had been introduced by PCR. A NotI fragment from plasmid pHSX-tetM-lacIOP containing the tetracycline-resistance gene (tet $M)$ and lac regulatory elements was inserted into the single Not I site of the construct created above to yield pVD300rec $A X_{\mathrm{Ec}}$ (Fig. 1).

Plasmid pEAS3 was constructed to allow simple, one-step, directional cloning of genes under control of lac regulatory elements for recombination into the recA locus of Gc. Plasmid pHSX-tetM-lacIOP was digested with EcoRI, and the ends were blunted with Klenow and ligated to a PacI linker that was digested with PacI and religated to create vector pEAS1. pEAS1 was subsequently digested with $B g l \mathrm{II}$, the ends were treated with mung bean nuclease, and the vector was religated to destroy the BglII site, yielding construct pEAS2. pEAS3 was created by inserting the NotI fragment from pEAS2, which contains the tetracycline-resistance gene (tetM) and lac regulatory elements, into the single NotI site of pVD300NL1.

The E. coli recA gene was amplified from plasmid pERA using primers ECRECAFORPAC (5'-GACTTTAATTAAAGGAGTAAAAATGGCTATC-3'), which introduces a PacI site (underlined), and ECRECAREVBGL (5'-AGTGAGATCT GATTCTGTCATGGCATATCC-3'), which introduces a $B g l I I$ site (underlined) by PCR using $P f u$ polymerase. To construct pBLUNT/EcrecA, the $1 \cdot 1 \mathrm{~kb}$ PCR fragment was gel purified and cloned into pCR-Blunt (Invitrogen), and sequenced to verify that no errors had been introduced by PCR. To construct pEAS $3 /$ rec $A_{\mathrm{Ec}}$ the $1.1 \mathrm{~kb}$ EcrecA-containing fragment was released from pBLUNT/EcrecA with a PacI/ $B g l$ II double digest, gel purified, and ligated to $\mathrm{PacI} / \mathrm{BglII}$ digested and gel-purified pEAS3, replacing the Gc recA with the E. coli recA. Therefore, apart from the insert, the only difference between plasmids pVD300rec $A X_{\mathrm{Ec}}$ and $\mathrm{pEAS} 3 / \mathrm{rec} A_{\mathrm{Ec}}$ is the multiple cloning site into which the insert is cloned (Fig. 1).

Plasmid constructs pVD300rec $A X_{\mathrm{Ec}}$ and $\mathrm{pEAS3} / \mathrm{rec} A_{\mathrm{Ec}}$ were recombined into the Gc chromosome at the recA locus, replacing the wild-type allele with $E$. coli recA gene alone or the $\mathrm{rec} A / \mathrm{rec} X$ locus, both under control of lac regulatory elements (Fig. 1). Gc strain FA1090 1-81-S2 was transformed with each plasmid by co-culture of plasmid DNA and Gc on solid media (spot transformation). Briefly, FA1090 was plated onto GCB and 20-40 $\mu \mathrm{g}$ of plasmid DNA was spotted onto individual areas of the plate. After co-incubation for 18-22 h, colonies from the spotted areas were collected with a Dacron swab, resuspended in GCBL and plated on GCB-Tet to select for transformants. Transformants were screened by Southern analysis of ClaI-digested chromosomal DNA with recA- and tet-specific probes (data not shown).

Construction of plasmid pGCC4/Ecrec $X$ and expression from the gonococcal Cg8 locus. The $E$. coli rec $X$ gene was amplified from plasmid pERA using primers ECRECXFORPAC $\left(5^{\prime}-\right.$ GTAGGTTAATTAAGTTGTAAGGATATGCCATGAC$3^{\prime}$ ), which introduces a PacI site (underlined), and ECRECXREV (5'-AGTCGCTAGCAATACCGTATGCGTTCAGTCG-3') by PCR using $P f u$ polymerase. pGCC4/ Ecrec $X$ was constructed by gel purifying the $0.5 \mathrm{~kb}$ recX PCR product and cloning it into PacI/PmeI-digested pGCC4. A single clone was sequenced to verify that no errors had been introduced by PCR. Strain FA1090rec $A_{\mathrm{Ec}}$ was transformed with pGCC4/EcrecX by spot transformation as 
described above. Transformants were selected on GCBErm and screened by PCR using primers LACPFOR $\left(5^{\prime}-\right.$ GAGCGGATAACAATTTCACA- $3^{\prime}$ ) and ECRECXREV to demonstrate insertion in the genome. To verify insertion into the proper chromosomal locus, a Southern blot analysis of ClaI-digested genomic DNA isolated from FA1090rec $A_{\mathrm{Ec}}$ and FA1090 rec $A_{\mathrm{Ec}} / \mathrm{rec} X_{\mathrm{Ec}}$ was performed using recX- and ermspecific probes (data not shown).

Genetic transfer in E. coli and Gc. P1 transduction and Hfr conjugation were performed as described by Miller (1992). For transduction, recipient strains of LB1, carrying one of the indicated plasmids, were grown for $4 \mathrm{~h}$ with $1.5 \mathrm{mM}$ IPTG induction before adding P1 phage grown on Hfr Cavalli (Bachmann, 1972). $\mathrm{Leu}^{+}$or Pro ${ }^{+}$transductants were selected in $2.5 \mathrm{ml}$ top agar on glucose minimal medium. For conjugation, overnight cultures of recipient strain LB1 carrying one of the indicated plasmids were diluted 1:10, the donor strain $(\mathrm{Hfr}$ Cavalli) was diluted 1:50, and both were grown for $4 \mathrm{~h}$ with $1.5 \mathrm{mM}$ IPTG. After normalizing by $\mathrm{OD}_{600}$, mating was performed at 1:5 donor to recipient for $3.5 \mathrm{~h}$. After washing with PBS, $\mathrm{Leu}^{+}$or $\mathrm{Pro}^{+}$transconjugant colonies were selected on glucose minimal agar. Donor c.f.u. were selected on glucose minimal agar with methionine. Recipient colonies were selected on LB agar with $50 \mu \mathrm{g}$ streptomycin $\mathrm{ml}^{-1}$.

Gonococcal transformation assays were performed as described previously (Stohl \& Seifert, 2001), and transformation efficiency was expressed as the number of $S p c^{R}$ transformants per c.f.u.

UV and nalidixic acid resistance assays. To measure UV resistance in E. coli, overnight cultures of each strain were grown for $4 \mathrm{~h}$ with $1.5 \mathrm{mM}$ IPTG, serially diluted, and spotdilutions were plated onto LB agar with $1.5 \mathrm{mM}$ IPTG. Plates were exposed to $1,2 \cdot 5,5 \cdot 0,7 \cdot 5$ and $10 \mathrm{~J} \mathrm{~m}^{-2} \mathrm{UV}$ light. Relative survival at each dose was calculated by comparing the number of c.f.u. in the irradiated sample to the number of c.f.u. in the non-irradiated sample. UV resistance of Gc was measured as previously described (Stohl \& Seifert, 2001).

Nalidixic acid causes DNA double-strand breaks (Drlica \& Zhao, 1997), and resistance to nalidixic acid exposure can be used to assess defects in DNA repair (McDaniel et al., 1978). To measure nalidixic acid resistance in E. coli, 1:10 dilutions of overnight cultures of each E. coli strain were grown for $4 \mathrm{~h}$ with $1.5 \mathrm{mM}$ IPTG and plated on LB agar with $15 \mu \mathrm{g} \mathrm{ml}^{-1}$ nalidixic acid. Total c.f.u. were calculated by plating spot dilutions of the cultures onto LB agar. Colonies were counted after $36 \mathrm{~h}$ growth, and resistance to nalidixic acid was expressed as $\mathrm{Nal}^{\mathrm{R}}$ c.f.u. per total c.f.u. for each strain. Resistance to nalidixic acid in Gc was assayed as described by Stohl \& Seifert (2001). This assay is not a measure of mutation frequency (see Results).

Quantitative colony phase variation assay. Colony phase variation was assessed as described by Stohl \& Seifert (2001) by streaking four piliated $\left(\mathrm{P}^{+}\right)$colonies heavily onto GCB with $1 \mathrm{mM}$ IPTG, growing cells for $24 \mathrm{~h}$, collecting cells and plating on GCB. After 18-20 h growth, colonies with a domed colony morphology and defined edge were scored as piliated $\left(\mathrm{P}^{+}\right)$, whereas colonies with a flat morphology and a lessdefined edge were scored as non-piliated $\left(\mathrm{P}^{-}\right)$. Colony phase variation was expressed as the percentage of colonies exhibiting a non-piliated morphology.

Western blot analysis. Total Gc lysate was run on SDS-PAGE gels as described by Stohl \& Seifert (2001). Protein concentration was determined using the BCA protein assay (Pierce Chemicals). Gels were blotted to PVDF membrane (Millipore) as described by Stohl \& Seifert (2001) and developed according to the ECL Western blotting protocol (Amersham Life Science) with the modifications described by Stohl \& Seifert (2001). Primary anti-RecA antibodies (a gift of Michael Cox, University of Wisconsin-Madison) and secondary goat anti-rabbit IgG antibodies conjugated to horseradish peroxidase (Roche Pharmaceuticals) were diluted 1:5000 in blocking solution. Primary RecX anti-peptide antibodies, raised in rabbits against peptide DDSRFVARFIASRSRKG conjugated to MAP resin (Research Genetics), were diluted 1:2500 in blocking solution. For quantitative Western blot analyses, equal amounts of total protein from strains FA1090recAX $\mathrm{E}_{\mathrm{Ec}}$, FA1090rec $A_{\mathrm{Ec}}$ and FA1090rec $A_{\mathrm{Ec}} / \mathrm{rec} \mathrm{X}_{\mathrm{Ec}}$ were separated on SDS-PAGE gels and probed with anti-E. coli RecA or anti-E. coli RecX peptide antiserum. We used whole-band densitometry to compare protein levels of the strains as follows: dilutions of four independent samples of each strain were run on duplicate gels, the gels were scanned, and signals were quantified using ImageQuant software (Molecular Dynamics).

\section{RESULTS}

\section{Rationale}

We tested the ability of the Gc recA gene to complement an $E$. coli recA mutant and the ability of the E. coli recA gene to complement a $\mathrm{Gc}$ rec $A$ mutant. In both species, the heterologous recA was compared to the homologous recA expressed from identical, tandem lac-derived promoters and operators (tac and UV5) to prevent differences in transcriptional initiation or regulation from influencing expression levels. In E. coli, comparisons were also made to a vector-carrying recA mutant strain. We have previously shown that expression of Gc recA with this lac-derived regulatory system results in RecA-dependent phenotypes being dependent on IPTG in the growth medium (Seifert, 1997).

\section{Analysis of RecA-dependent phenotypes in Gc}

To examine the ability of the E. coli recA to mediate recombination processes in $\mathrm{Gc}$, we introduced the $E$. coli recA locus and the associated gene recX (Fig. 1), under control of lac regulatory signals, into the recA locus of Gc FA1090 variant 1-81-S2 by natural transformation $\left(\mathrm{FA} 1090 \mathrm{rec} A X_{\mathrm{Ec}}\right)$. We compared the ability of FA1090rec $A X_{\mathrm{Ec}}$ to mediate the recA-dependent phenotypes of DNA transformation, DNA repair and pilus antigenic variation to strain FA1090recA6, which has the Gc recA under control of the identical lac regulatory sequences, and to strain FA1090recA9, a recA null mutant that has $\operatorname{rec} A$ disrupted by an erm $C$ resistance cassette (Seifert, 1997) (Table 1). The DNA transformation efficiency of FA1090recAX $X_{\mathrm{Ec}}$ was significantly decreased relative to FA1090recA6, but much higher than FA1090recA9, suggesting that the recombinase activity of the E. coli RecA functions in Gc transformation, albeit not at wild-type levels (Fig. 2a).

DNA repair ability was assessed by measuring resistance to UV irradiation, which causes lesions in DNA, and resistance to nalidixic acid exposure, which introduces double-strand breaks into DNA (Drlica \& Zhao, 1997) 
Table 1. Complementation of RecA-mediated phenotypes

,$++/-,-$ and - - refer to full complementation, intermediate complementation, no complementation and lower than the null allele, respectively.

\begin{tabular}{|c|c|c|c|c|c|}
\hline \multicolumn{4}{|c|}{ RecA-mediated phenotypes in Gc } & \multicolumn{2}{|c|}{ RecA-mediated phenotypes in $E$. coli } \\
\hline $\operatorname{rec} A$ allele & $\begin{array}{c}\text { DNA } \\
\text { transformation }\end{array}$ & $\begin{array}{l}\text { DNA } \\
\text { repair }\end{array}$ & $\begin{array}{l}\text { Pilus antigenic } \\
\text { variation }\end{array}$ & $\begin{array}{l}\text { Genetic } \\
\text { transfer* }\end{array}$ & $\begin{array}{l}\text { DNA } \\
\text { repair }\end{array}$ \\
\hline Gct & + & + & + & + & $+/-$ \\
\hline E. coli $\neq$ & $+/-$ & -- & + & + & + \\
\hline Nulls & - & - & - & - & - \\
\hline
\end{tabular}

* As measured by P1 transduction and Hfr conjugation.

† Strain carries either recA6 or pVD300recA6.

$\neq$ Strain carries either $\operatorname{rec} A X_{\mathrm{Ec}}$ or $\mathrm{pVD} 300 \mathrm{rec} A X_{\mathrm{Ec}}$.

\Strain carries either recA9 or pACYC184.

(a)



(c)

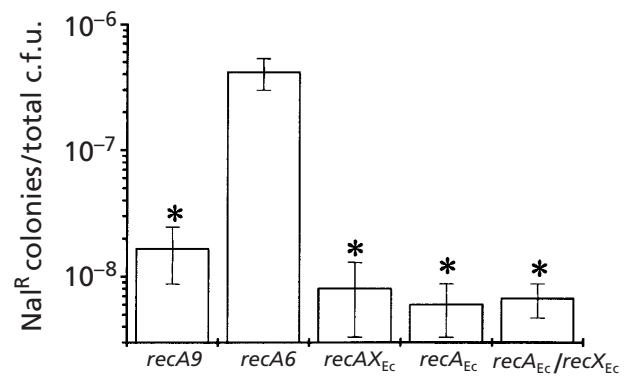

(b)

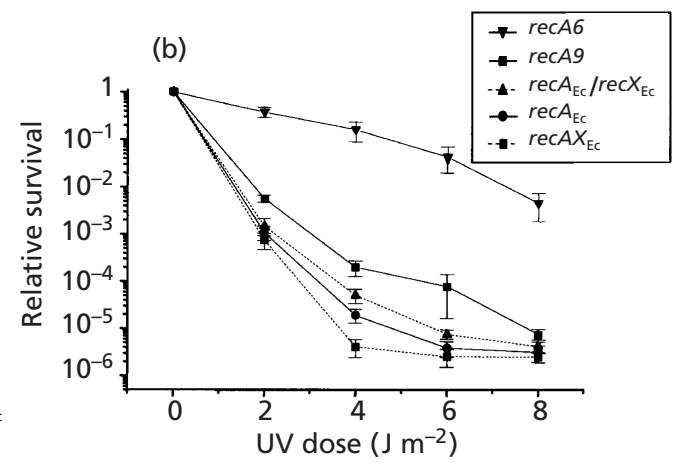

(d)

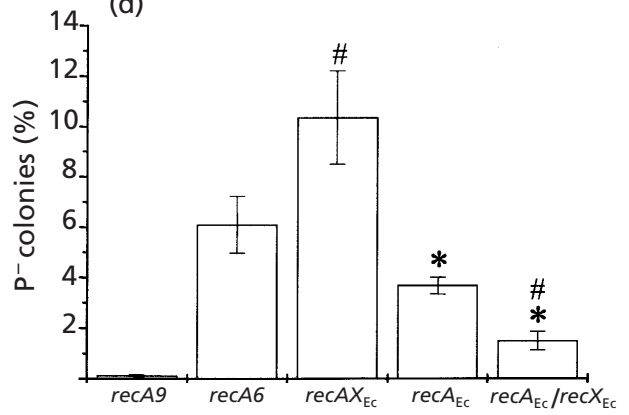

Fig. 2. RecA-mediated phenotypes of recA alleles expressed in Gc. (a) Efficiency of transformation to spectinomycin resistance $\left(S p c^{R}\right)$ by $S p c^{R}$ genomic DNA. Error bars represent the SEM of three experiments done in triplicate. ${ }^{*}, P<0.05$ compared to FA1090recA6 by Student's $t$-test; \#, $P<0.05$ compared to FA1090rec $A_{E d} / r e c X_{E c}$ by Student's $t$-test. The transformation efficiency for FA1090recA9 was $8.0 \times 10^{-7}$. (b) Relative survival after UV irradiation. Error bars represent the SEM of at least four experiments. At the $4 \mathrm{~J} \mathrm{~m}^{-2}$ dose the differences between all strains are statistically significant with $P<0.05$ by Student's $t$-test. (c) Relative survival on nalidixic acid. Error bars represent the SEM of two experiments done in duplicate. ${ }^{*}, P<0.05$ compared to FA1090recA6 by Student's $t$-test. (d) Percentage colony phase variation. Error bars represent the SEM of at least four independent experiments. *, $P<0.05$ compared to FA1090recAX $X_{\mathrm{Ec}}$ by Student's $t$-test; $\#, P<0.05$ compared to FA1090rec $A_{\mathrm{Ec}}$ by Student's $t$-test.

(Fig. 2b, c). Strain FA1090recAX ${ }_{\mathrm{Ec}}$ showed a level of UV resistance significantly lower than both FA1090recA6 and FA1090recA9, and the three strains showed a similar trend in nalidixic acid resistance. To verify that we were not simply measuring the rate of spontaneous mutation to nalidixic acid resistance, the effect of the different RecA proteins on frequency of spontaneous mutation to rifampin or spectinomycin resistance was determined. No significant difference in mutation rate was observed in strains carrying the E. coli or Gc recAs (data not shown).

Because of the decrease in resistance to UV light and nalidixic acid, we assumed that strain FA1090rec $A X_{\mathrm{Ec}}$ 
would also be deficient in pilin antigenic variation. Instead, it showed a slight, albeit not statistically significant, increase relative to FA1090recA6. To determine whether the increase in $\mathrm{P}^{-}$colony phase variants was due to an alteration of the mechanism of pilin antigenic variation, we analysed $\mathrm{P}^{-}$colony variants from FA1090recA6 and FA1090recAX $\mathrm{Ec}_{\mathrm{Ec}}$. Analysis of individually derived $\mathrm{P}^{-}$colony variants from strains FA1090recAX $\mathrm{Ec}_{\mathrm{Ec}}$ and FA1090recA6 showed a similar percentage resulting from pilE deletions $(29 \%$ in FA1090recAX $\mathrm{Ec}_{\mathrm{Ec}}$ vs $22 \%$ in FA1090recA6). Sequence analysis of the pilE genes of 10 independently derived, undeleted $\mathrm{P}^{-}$colony variants of the $\mathrm{FA} 1090$ rec $A X_{\mathrm{Ec}}$ or FA1090recA6 strains showed nearly identical repertoires of pilS copies as donors in these pilE variants (data not shown). Therefore, the increase in $\mathrm{P}^{-}$colony phase variants was not due to an alteration of the mechanism of pilin antigenic variation.

\section{RecA-dependent phenotypes mediated by $E$. coli recA alone and with recX in $\mathrm{Gc}$}

To determine the possible contribution of E. coli recX to the phenotypes we had observed, we constructed two additional strains of Gc. Strain FA1090 rec $A_{\mathrm{Ec}}$ has the $E$. coli recA gene alone under lac regulatory signals introduced into the recA locus of FA1090 by natural DNA transformation, and strain FA1090 rec $A_{\mathrm{Ec}} / \mathrm{rec}_{\mathrm{Ec}}$ is strain FA1090rec $A_{\mathrm{Ec}}$ with the E. coli recX introduced into an unlinked chromosomal locus $(\mathrm{Cg} 8)$ by natural transformation, also under control of lac regulatory sequences. The $\mathrm{Cg} 8$ locus is a transcriptionally inactive region of the FA1090 chromosome determined to not play a role in recombination (Mehr et al., 2000). In all strains of Gc used in these analyses there is a chromosomal copy of the Gc recX; however, since it is present in all strains, it does not affect the interpretation of data.

There was a consistent trend in the efficiencies of RecAmediated processes among the Gc strains containing $E$. coli recA genes (Fig. 2). Strain FA1090rec $A X_{\mathrm{Ec}}$ showed the highest levels of DNA transformation and colony phase variation, while $\mathrm{FA} 1090 \mathrm{rec} \mathrm{A}_{\mathrm{Ec}} /$ rec $\mathrm{X}_{\mathrm{Ec}}$ showed a statistically significant decrease relative to FA1090recAX $\mathrm{Ec}_{\mathrm{Ec}}$ in both DNA transformation and colony phase variation. Strain FA1090rec $A_{\mathrm{Ec}}$ showed an intermediate level of DNA transformation ability, albeit statistically indistinguishable from FA1090recAX $\mathrm{Ec}_{\mathrm{Ec}}$, and an intermediate level of colony phase variation, statistically distinct from both FA1090recAX $X_{\mathrm{Ec}}$ and $\mathrm{FA} 1090$ rec $A_{\mathrm{Ec}} / \mathrm{rec} \mathrm{X}_{\mathrm{Ec}}$. The differences in UV resistance followed exactly the opposite trend, with FA1090recAX $X_{\mathrm{Ec}}$ showing the lowest level of UV resistance and FA1090rec $A_{\mathrm{Ec}} / \mathrm{rec} X_{\mathrm{Ec}}$ showing the highest UV resistance. Additionally, all the Gc strains containing E. coli recA genes showed slightly lower, but statistically the same, levels of resistance to nalidixic acid than the recA mutant strain, FA1090recA9.
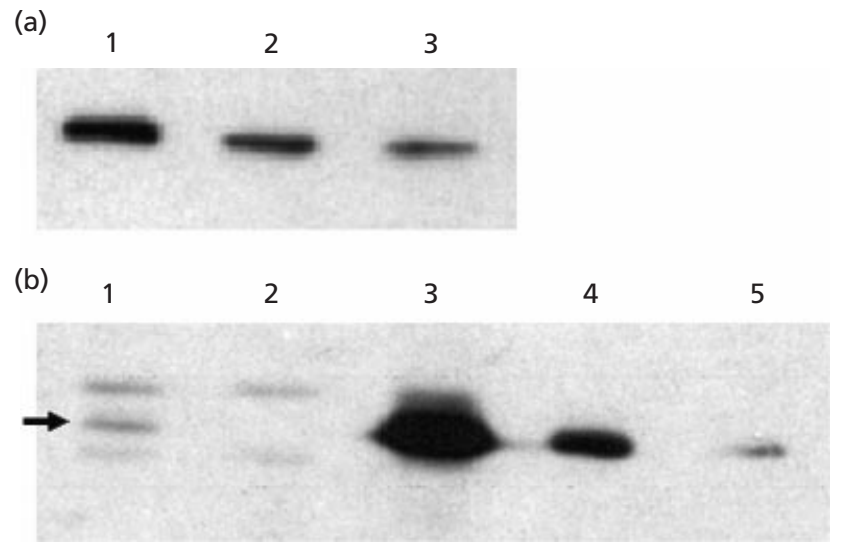

Fig. 3. Representative Western blot analyses of RecA or RecX protein levels. (a) SDS-PAGE gels containing $0.1 \mu \mathrm{g}$ total protein per lane of strains FA1090recAX $X_{E C^{\prime}}, F A 1090 r e c A_{E c}$ and $\mathrm{FA} 1090 \mathrm{rec} A_{\mathrm{EC}} / \mathrm{rec} X_{\mathrm{EC}}$ (lanes 1, 2 and 3, respectively) were run and transferred to PVDF membrane, with subsequent Western blot analysis using anti-RecA antisera for detection with the ECL detection kit. (b) SDS-PAGE gels containing $1 \mu \mathrm{g}$ total protein per lane of strains FA1090recAX $X_{E C}, F A 1090 r e c A_{E C}$ and FA1090rec $A_{E C} / r e c X_{E C}$ (lanes 1, 2 and 3, respectively), or 0.1 or $0.02 \mu \mathrm{g}$ of $\mathrm{FA} 1090 \mathrm{rec} A_{\mathrm{EC}} / \mathrm{rec} X_{\mathrm{EC}}$ (lanes 4 and 5) were run and transferred to PVDF membrane, with subsequent Western blot analysis using anti-E. coli RecX peptide antisera for detection with the ECL detection kit. The arrow indicates the mobility of the RecX protein.

\section{E. coli RecA protein levels in strains of Gc correlate with RecA-mediated phenotypes}

The three Gc strains containing E. coli recA genes exhibited differential RecA-mediated phenotypes in Gc, and the E. coli recX gene is present in two of these strains, FA1090recAX $\mathrm{Ec}_{\mathrm{Ec}}$ and $\mathrm{FA1090rec} \mathrm{A}_{\mathrm{Ec}} / \mathrm{recX}_{\mathrm{Ec}}$. Studies of recX in other organisms have suggested that recX may play a role in downregulating recA expression (Sano, 1993; Vierling et al., 2000). We therefore wanted to determine whether RecA protein levels were influenced by the presence of $r e c X$ and whether the level of RecA protein reflected the abilities of the strains to carry out recombination-associated processes. Quantitative Western blot analysis was performed to determine levels of RecA protein (see Methods). These results show that the levels of RecA protein are highest in strain FA1090rec $A X_{\mathrm{Ec}}$ and lowest in FA1090rec $A_{\mathrm{Ec}} /$ rec $X_{\mathrm{Ec}}$, with FA1090rec $A_{\mathrm{Ec}}$ showing an intermediate level of $E$. coli RecA protein (Fig. 3a). Values obtained were used to express the level of RecA as a percentage of the strain expressing the highest level of RecA, FA1090recAX $\mathrm{Ec}$. Strain FA1090rec $A_{\mathrm{Ec}}$ expressed $76 \%( \pm 0.04$ standard error) the RecA protein of $\mathrm{FA1090recAX} \mathrm{Ec}_{\mathrm{Ec}}$, and FA1090rec $A_{\mathrm{Ec}} /$ rec $\mathrm{X}_{\mathrm{Ec}}$ expressed $54 \%( \pm 0.05$ standard error) the RecA protein of FA1090rec $A X_{\mathrm{Ec}}$. Therefore, the level of RecA protein in these strains correlates directly with the ability of the strains to carry out DNA transformation and colony phase variation, and inversely with resistance to DNA-damaging agents. 
(a)

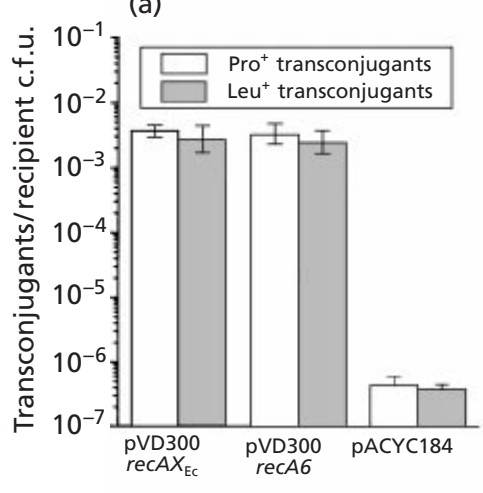

(c)



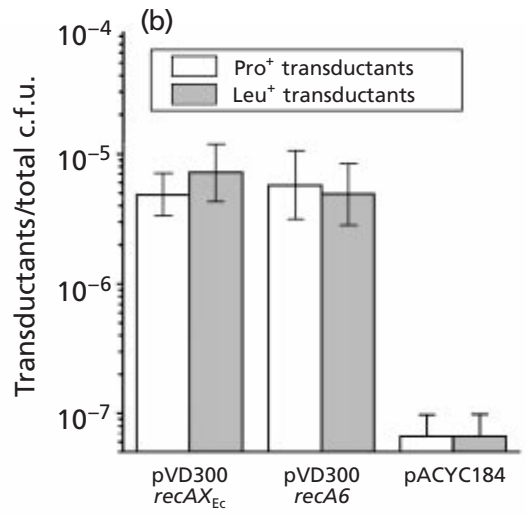

(d)

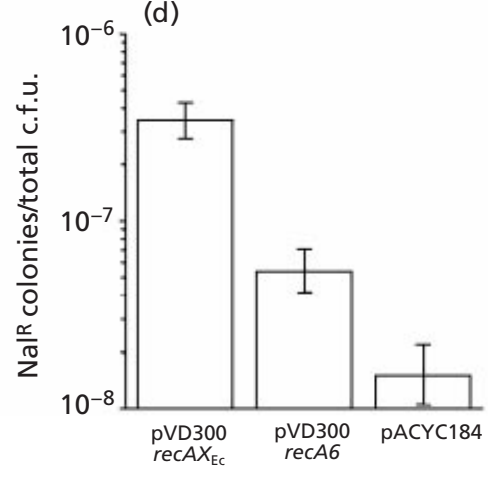

Fig. 4. RecA-mediated phenotypes of recA alleles expressed in $E$. coli. Assays were done with strain LB1, a $\triangle$ recA derivative of AB1157, except when noted. Error bars represent the SEM of three independent experiments. (a) Hfr conjugation of proline or leucine prototrophy from strain Hfr Cavalli to strain LB1 carrying the indicated plasmids. (b) P1 transduction of proline or leucine prototrophy from strain Hfr Cavalli to strain LB1 carrying the indicated plasmids. (c) Relative survival after UV irradiation of strains LB1 or LB2, a $\triangle$ recA derivative of DM49 (AB1157lexA3), carrying the indicated plasmids: $\boldsymbol{\square}, \mathrm{LB} 1\left(\mathrm{pVD} 300 \mathrm{rec} A X_{\mathrm{EC}}\right) ; \square, \mathrm{LB} 2\left(\mathrm{pVD} 300 \mathrm{rec} A X_{\mathrm{EC}}\right)$; $\boldsymbol{0}, \mathrm{LB} 1(\mathrm{pVD} 300 \mathrm{rec} A 6) ; 0, \mathrm{LB} 2(\mathrm{pVD} 300 \mathrm{rec} A 6) ; \boldsymbol{\Delta}$ LB1(pACYC184); $\triangle$, LB2(pACYC184). (d) Relative survival on nalidixic acid of strain LB1 carrying the indicated plasmids.

\section{Overexpression of $E$. coli recX downregulates $E$. coli RecA in Gc}

Since RecA protein levels may be influenced by $\operatorname{rec} X$, we wanted to determine the relative level of E. coli recX expression in strains FA1090recAX $\mathrm{Ec}_{\mathrm{E}}$ and FA1090rec $A_{\mathrm{Ec}} /$ rec $\mathrm{X}_{\mathrm{Ec}}$. In strain FA1090recAX $\mathrm{Ec}_{\mathrm{cc}}$, recX is found directly downstream of $r e c A$ on the same DNA fragment, and in strain FA1090rec $A_{\mathrm{Ec}} / \mathrm{rec} \mathrm{X}_{\mathrm{Ec}}, \operatorname{rec} \mathrm{X}$ is expressed from a different locus of the Gc chromosome, but in both strains recX is under control of lac regulatory signals. Western blot analysis was performed as described above, except using an anti-E. coli RecX

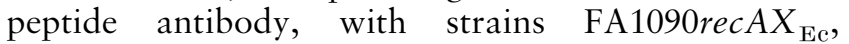
FA1090rec $A_{\mathrm{Ec}} / \mathrm{rec} \mathrm{X}_{\mathrm{Ec}}$ and $\mathrm{FA1090rec} A_{\mathrm{Ec}}$ (a negative control that does not contain the $E$. coli recX). Strain FA1090rec $A_{\mathrm{Ec}} / \mathrm{rec} \mathrm{X}_{\mathrm{Ec}}$ was found to have RecX levels over 50-fold higher than FA1090recAX $\mathrm{Ec}_{\mathrm{Ec}}$ (Fig. 3b). Similarly, recX transcript levels were found to be approximately 10-fold higher in $\mathrm{FA1090rec} \mathrm{A}_{\mathrm{Ec}} / \mathrm{recX}_{\mathrm{Ec}}$ than in FA1090rec $A X_{\mathrm{Ec}}$ (data not shown), demonstrating that overexpression of $r e c X$ results in decreased RecA levels and thereby affects RecA-mediated phenotypes.

\section{Analysis of RecA-dependent phenotypes in E. coli}

We were intrigued by the differential recombination phenotypes observed when the E. coli recA was expressed in Gc, so we tested the ability of the Gc RecA to mediate recombination processes in E. coli (Table 1). $\Delta r e c A$ derivatives of AB1157 (DeWitt \& Adelberg, 1962), LB1 and LB2, were made by P1 transduction (see Methods). LB2 additionally contains the lexA3 mutation (Mount et al., 1972). A low-copy-number derivative of pACYC184 (Chang \& Cohen, 1978), with an estimated copy number of 10 , was used to express the Gc recA gene under IPTG control (pVD300recA6) in E. coli. This plasmid was compared to an identical plasmid carrying the E. coli recA/recX operon under IPTG control $\left(\mathrm{pVD} 300 \mathrm{rec} A X_{\mathrm{Ec}}\right)$, where $76 \mathrm{bp}$ separates the stop codon of recA from the start codon of recX. In this construct, both recA and recX are present at the same copy number and under control of the same lac promoter as the Gc recA.

The chromosomal copy of E. coli recX is still present in strains LB1 and LB2; however, two lines of evidence suggest that the level of expression of chromosomal 
recX is negligible and should not alter the interpretation of subsequent assays. First, we were not able to detect RecX protein expression by Western blot analysis in either strain AB1157 or strain JC10289, the parental strains of LB1 and LB2 (data not shown). Second, repeated RT-PCR experiments on strain AB1157 showed a barely detectable level of recX RT-PCR product (data not shown). Thus, comparison of the RecA-mediated phenotypes of DNA repair and homologous recombination in the $\Delta r e c A$ strains transformed with the three low-copy plasmids pACYC184, $\mathrm{pVD} 300$ rec $A 6$ and $\mathrm{pVD} 300$ rec $A X_{\mathrm{Ec}}$ is not likely to be affected by the chromosomal copy of recX.

The lac-regulated, plasmid-expressed Gc and E. coli RecAs mediated nearly identical levels of homologous recombination in LB1 as measured by P1 phagemediated transduction and Hfr conjugation, that were significantly higher than the LB1 vector control (Fig. 4a, b). These results indicate that these RecA homologues mediate homologous recombination with similar efficiencies in E. coli.

Resistance to UV light and nalidixic acid, which causes double-strand breaks and is used to assess defects in DNA repair (McDaniel et al., 1978), were used to measure DNA repair capabilities of the RecA proteins in strain LB1 (Fig. 4c, d), which is able to activate the SOS response. The UV resistance of the LB1 pVD300recA6 strain was 2000-3000-fold lower than that of the LB1 $\mathrm{pVD} 300 \mathrm{rec} A X_{\mathrm{Ec}}$ strain, but both strains showed higher levels of resistance than the vector control. Similar differences between the LB1 pVD300recA6 and $\mathrm{pVD} 300 \mathrm{rec} A X_{\mathrm{Ec}}$ strains were also observed when resistance to nalidixic acid was measured by direct plating (Fig. 4d). An additional assay was performed to assess resistance to nalidixic acid in which LB1 cells carrying the three plasmids were grown in liquid culture, exposed to nalidixic acid for increasing amounts of time, and subsequently plated on LB without the antibiotic. We noted the same trend in survival as observed with the direct-plating method, with LB1 pVD300recAX $X_{\mathrm{Ec}}$ showing slightly higher survival than the same strain carrying pVD300recA6, and pACYC184 showing much lower survival than the two recA-containing strains. Subsequent analysis of 100 of the surviving colonies from each strain revealed that all the colonies that had been exposed to nalidixic acid remained $\mathrm{Nal}^{\mathrm{S}}$, demonstrating that this assay is not a measure of mutation frequency (data not shown).

Repair of UV-induced DNA lesions in E. coli involves induction of the SOS response. Since Gc does not have an SOS system (Black et al., 1998), we wanted to test the hypothesis that the inability of the Gc RecA to induce the SOS response was responsible for the inferred inefficient DNA repair. To do this, we used two experimental approaches. First, we measured the UV resistance of strain LB2, a lexA3 mutant with an uncleavable repressor unable to induce the SOS response, carrying plasmids pVD300recA6 or pVD300rec $A X_{\mathrm{Ec}}$ (Fig. 4c). The difference in survival between LB2 pVD300recA6 and LB2 pVD300recAX $\mathrm{Ec}_{\mathrm{c}}$ was reduced compared to that seen in the LB1 strains; however, the pVD300recA6 strain was still reduced in survival relative to the $\mathrm{pVD} 300 \mathrm{rec} A X_{\mathrm{Ec}}$ strain (1-2 log values lower). Second, strain LB1 carrying a chromosomal dinD1:Mud:lacZ (Kenyon \& Walker, 1980) fusion was transformed with plasmids $\mathrm{pVD} 300 \mathrm{rec} A X_{\mathrm{Ec}}$ or $\mathrm{pVD} 300$ rec A6. The LB1 strain carrying pVD300recA6 showed minimal induction of $\operatorname{din} D 1$ expression relative to the LB1 strain carrying $\mathrm{pVD} 300$ rec $A X_{\mathrm{Ec}}$ after exposure to mitomycin $\mathrm{C}$, an SOS-inducing signal (data not shown). Therefore, the Gc RecA inefficiently mediates DNA repair in E. coli, and this is partly due to an inability to induce the SOS response.

\section{DISCUSSION}

We tested the abilities of the Gc and E. coli RecAs to mediate the major forms of genetic transfer in E. coli (transduction and conjugation) and Gc (DNA transformation). In both bacteria these genetic processes share a dependency upon the RecBCD pathway of homologous recombination, although the RecBCD dependency of conjugation in E. coli (Kushner et al., 1971; Lloyd \& Thomas, 1983) is more stringent than the RecBCD dependency of DNA transformation in Gc (Mehr \& Seifert, 1998). The RecA and RecBCD proteins from $E$. coli have been shown to interact in vitro (Amundsen et al., 2000; Churchill et al., 1999). Previous work by De Vries \& Wackernagel (1992) suggests that RecA and RecBCD proteins from different species of bacteria do not interact as efficiently as proteins from the same species of bacteria in vivo. In these studies, researchers measured the ability of the RecA proteins from Serratia marcescens and Proteus mirabilis, both enterobacteria closely related to $E$. coli, to mediate conjugal recombination in E. coli. Although the $S$. marcescens RecA was able to catalyse recombination levels equal to the E. coli RecA, the P. mirabilis RecA was only $50 \%$ as effective, suggesting that the protein from $P$. mirabilis did not interact as efficiently with the RecBCD enzyme from E. coli. To further study the interactions of the RecA and RecBCD proteins from these bacteria, all possible combinations of $\operatorname{rec} A$ and recBCD genes from $P$. mirabilis, S. marcescens and E. coli were expressed in E. coli and tested for recombination. Several of the interspecies combinations failed to reach the level of the intraspecies combinations, further suggesting that decreases in recombination were due to inefficient interactions of the RecA and RecBCD enzymes. In our experiments expressing the Gc and $E$. coli recA genes in E. coli, DNA transduction and conjugation were mediated with equal efficiency by both RecA proteins, suggesting that the proteins shared similar recombination activities and that the Gc RecA was able to interact as efficiently as the E. coli RecA with the E. coli RecBCD enzyme. The major form of genetic transfer in Gc (DNA transformation) was mediated with decreased efficiency by all the strains carrying $E$. coli recA, independent of $r e c X$. These results imply that the E. coli RecA and the Gc RecBCD interact with decreased efficiency. Alternatively, since $\operatorname{rec} B C D \mathrm{mu}-$ 


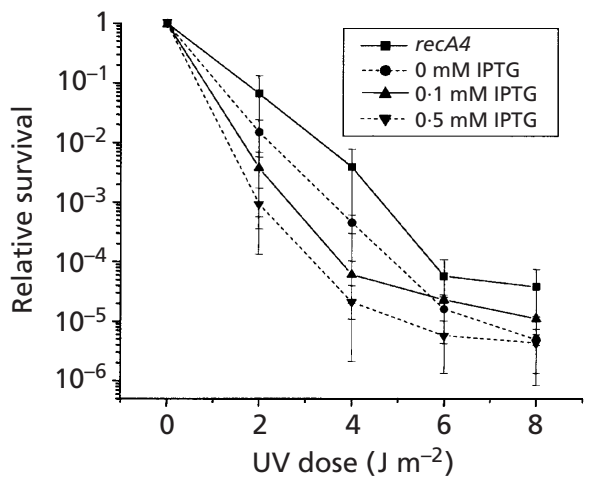

Fig. 5. Relative UV survival of $F A 1090 r e c A_{E C}$ expressing varying levels of RecA. Error bars represent the SEM of at least two experiments. Strain recA4 is a recA null allele identical to strain FA1090recA9, except that it carries a tet rather that an erm resistance cassette (Seifert, 1997).

tants in Gc still show substantial transformation ability (Mehr \& Seifert, 1998), there is likely to be RecBCDindependent recombination that functions in natural DNA transformation, and the E. coli RecA may be deficient in mediating recombination via this second pathway.

Expression of the heterologous RecA protein from either bacterium did not confer as high a resistance to DNAdamaging agents as expression of the homologous RecA protein. In E. coli, the Gc RecA only partially complemented for survival to DNA-damaging agents; this may be due to a reduced ability of this RecA protein to induce the SOS response. Since there is no SOS-like system in Gc (Black et al., 1998), the Gc recA may not be adapted to induce a full SOS response. The pVD300recA6 construct also lacks a LexA-binding site in the promoter region (Fyfe \& Davies, 1990), and there should be no further induction of the lac-driven promoters during SOS. Furthermore, one outcome of SOS induction in E. coli is RecA-assisted cleavage of UmuD, a protein that functions in UV-induced mutagenesis (Walker, 1996). Since there is no UmuD homologue in Gc (Roe et al., 1997), the Gc RecA may not be able to activate this cellular response, resulting in decreased resistance to DNA damage. recA genes from many bacteria have been shown to only partially complement E. coli recA mutants for UV resistance (Miller \& Kokjohn, 1990). Specifically, expression of the recA gene from Bacteriodes fragilis in an $E$. coli recA mutant revealed the same inefficient complementation for UV resistance as we observed. Interestingly, B. fragilis also lacks an error-prone DNA repair system (Goodman et al., 1987). A far more dramatic difference in DNA repair was observed in Gc. Strains of Gc carrying E. coli recA were actually less resistant to the DNA-damaging agents UV light and nalidixic acid than a recA null mutant. This markedly decreased resistance was influenced by levels of E. coli RecA protein, but was not simply a result of overexpression of the E. coli RecA. In the three Gc strains containing E. coli recA genes, the strain with the highest level of E. coli RecA protein, FA1090recAX $\mathrm{Ec}$, was least resistant to UV irradiation, whereas the strain with the lowest amount of RecA protein, FA1090rec $A_{\mathrm{Ec}} / \mathrm{rec} X$, was the most resistant. In a series of UV resistance assays using strain FA1090rec $A_{\mathrm{Ec}}$, which has recA under tight control of lac regulatory signals, increasing the level of IPTG in the medium, thereby increasing RecA levels, resulted in decreased UV resistance (Fig. 5). However, even when levels of IPTG were titrated to zero, strain FA1090 rec $A_{\mathrm{Ec}}$ was still less resistant to UV irradiation than a recA mutant strain (Fig. 5). It seems possible that, even when present in small quantities in Gc, the E. coli RecA inhibits some protein involved in DNA repair or interacts with damaged DNA in such a way that it interferes with DNA repair. Although expression of the recA gene from Acholeplasma laidlawii, a mycoplasma, is lethal in E. coli (Dybvig \& Zhao, 1997), as far as we are aware this is the first published example of a specific hypersensitivity to UV light conferred by expression of a heterologous recA gene.

Since the Gc and E. coli RecA proteins differed in their abilities to mediate various RecA-dependent processes in the heterologous host, and since the carboxy-terminal portion of the two RecA proteins is the most variable, we wanted to determine whether we could correlate the phenotypic differences with specific regions of the two RecA proteins. To do this, we created tripartite hybrid RecA molecules of the E. coli and Gc RecA proteins where the $\mathrm{N}$-terminal, central and $\mathrm{C}$-terminal portions of the two proteins were arranged in all possible combinations, and assayed these proteins for the ability to carry out RecA-mediated processes in E. coli and Gc. We found that activity of the RecA proteins did not segregate to a particular domain, suggesting that many regions of the RecA protein are important for activity (L. Blount \& H. S. Seifert, unpublished data).

recX genes have been identified downstream of $\operatorname{rec} A$ in many bacteria by sequence analysis (De Mot et al., 1994). Work in Pseudomonas aeruginosa, Mycobacterium smegmatis and Streptomyces lividans has demonstrated that overexpression of recA from a plasmid construct is deleterious or lethal in the absence of recX (Papavinasasundaram et al., 1997; Sano, 1993; Vierling et al., 2000), leading to the hypothesis that RecX acts by downregulating expression of recA or activity of the RecA protein. However, this hypothesis has not been rigorously tested, and a recX mutation of $S$. lividans does not affect any RecA-mediated processes or RecA levels (Vierling et al., 2000). Gc also contains a recX homologue; however, it is not located downstream of recA. Furthermore, inactivation of recX in Gc impairs the RecA-mediated processes of antigenic variation, DNA transformation and DNA repair, but does not affect RecA protein levels (Stohl \& Seifert, 2001), demonstrating that RecX functions differently in certain species of bacteria. All the strains of Gc used in these experiments contain a functional Gc recX; however, since it is present in all strains, it is not responsible for 
the differences in observed phenotypes, and we do not know if it can interact with E. coli RecA.

Our current data demonstrate that the E. coli RecX influences RecA-mediated processes; however, E. coli RecX acts by modulating E. coli RecA levels in a complex manner. Comparing RecA protein levels in strains FA1090rec $A_{\mathrm{Ec}}$ and FA1090rec $A_{\mathrm{Ec}} /$ rec $\mathrm{X}_{\mathrm{Ec}}$ demonstrated that overexpressing RecX results in decreased RecA levels and corresponding effects on RecA-related phenotypes. However, strain FA1090rec $A X_{\mathrm{Ec}}$ shows the highest RecA protein levels and RecA-related phenotypes relative to FA1090rec $A_{\mathrm{Ec}}$, but expresses about 50 times less RecX than FA1090rec $A_{\mathrm{Ec}} / \mathrm{rec} \mathrm{X}_{\mathrm{Ec}}$. These data suggest that RecX (or $r e c X$ ) may perform an additional function of either stabilizing the recA transcript, stabilizing the RecA protein, or somehow enhancing RecA activity when present either at low levels or when present on the same transcript as recA. Therefore, the apparent biological role of E. coli RecX may be dependent upon the level of the RecX present in a cell, with lower levels of RecX activating or positively regulating RecA, but higher levels of the RecX protein inhibiting or downregulating RecA. Studies of the E. coli $r e c X$ in Gc have stimulated thoughts about the function of RecX, and we are currently conducting experiments to determine the role of this protein in DNA recombination and repair in E. coli.

\section{ACKNOWLEDGEMENTS}

We thank Deborah Tobiason and Eric Skaar for editorial suggestions for the manuscript, and Mike Cox for supplying RecA antiserum. This work was supported by NIH grant RO1 AI44239. Elizabeth A. Stohl was partially supported by ACS postdoctoral fellowship \#PF-00-016-GMC and NIH training grant T32AI07476.

\section{REFERENCES}

Amundsen, S. K., Taylor, A. F. \& Smith, G. R. (2000). The RecD subunit of the Escherichia coli RecBCD enzyme inhibits RecA loading, homologous recombination, and DNA repair. Proc Natl Acad Sci U S A 97, 7399-7404.

Bachmann, B. J. (1972). Pedigrees of some mutant strains of Escherichia coli K-12. Bacteriol Rev 36, 525-557.

Black, C. G., Fyfe, J. A. M. \& Davies, J. K. (1998). Absence of an SOS-like system in Neisseria gonorrhoeae. Gene 208, 61-66.

Chang, A. C. \& Cohen, S. N. (1978). Construction and characterization of amplifiable multicopy DNA cloning vehicles derived from the P15A cryptic miniplasmid. J Bacteriol 134, 1141-1156.

Chaussee, M. S., Wilson, J. \& Hill, S. A. (1999). Characterization of the recD gene of Neisseria gonorrhoeae MS11 and the effect of recD inactivation on pilin variation and DNA transformation. Microbiology 145, 389-400.

Churchill, J. J., Anderson, D. G. \& Kowalczykowski, S. C. (1999). The RecBC enzyme loads RecA protein onto ssDNA asymmetrically and independently of chi, resulting in constitutive recombination activation. Genes Dev 13, 901-911.

Cox, M. M. (1993). Relating biochemistry to biology: how the recombinational repair function of RecA protein is manifested in its molecular properties. Bioessays 15, 617-623.
Csonka, L. N. \& Clark, A. J. (1979). Deletions generated by the transposon $\mathrm{Tn} 10$ in the srl recA region of the Escherichia coli K-12 chromosome. Genetics 93, 321-343.

De Mot, R., Schoofs, G. \& Vanderleyden, J. (1994). A putative regulatory gene downstream of recA is conserved in Gramnegative and Gram-positive bacteria. Nucleic Acids Res 22, 1313-1314.

De Vries, J. \& Wackernagel, W. (1992). Recombination and UV resistance of Escherichia coli with the cloned recA and recBCD genes of Serratia marcescens and Proteus mirabilis: evidence for an advantage of intraspecies combination of $P$. mirabilis RecA protein and RecBCD enzyme. J Gen Microbiol 138, 31-38.

DeWitt, S. K. \& Adelberg, E. A. (1962). The occurrence of a genetic transposition in a strain of E. coli. Genetics 47, 577-585. Drlica, K. \& Zhao, X. (1997). DNA gyrase, topoisomerase IV, and the 4-quinolones. Microbiol Mol Biol Rev 61, 377-392.

Dybvig, K. \& Zhao, X. (1997). Cloning and DNA sequence of a mycoplasmal recA gene. J Bacteriol 174, 778-784.

Fyfe, J. A. \& Davies, J. K. (1990). Nucleotide sequence and expression in Escherichia coli of the recA gene of Neisseria gonorrhoeae. Gene 93, 151-156.

Goodman, H. J., Parker, J. R., Southern, J. A. \& Woods, D. R. (1987). Cloning and expression in Escherichia coli of a recA-like gene from Bacteroides fragilis. Gene 58, 265-271.

Haas, R. \& Meyer, T. F. (1986). The repertoire of silent pilus genes in Neisseria gonorrhoeae: evidence for gene conversion. Cell 44, 107-115.

Hagblom, P., Segal, E., Billyard, E. \& So, M. (1985). Intragenic recombination leads to pilus antigenic variation in Neisseria gonorrhoeae. Nature 315, 156-158.

Hill, S. A. (2000). Neisseria gonorrhoeae recJ mutants show defects in recombinational repair of alkylated bases and UVinduced pyrimidine dimers. Mol Gen Genet 264, 268-275.

Hill, S. A. \& Grant, C. C. R. (2002). Recombinational error and deletion formation in Neisseria gonorrhoeae: a role for RecJ in the production of $p_{i l E_{L}}$ deletions. Mol Genet Genomics 266, 962-972.

Howell-Adams, B. \& Seifert, H. S. (2000). Molecular models accounting for the gene conversion reactions mediating gonococcal pilin antigenic variation. Mol Microbiol 22, 509-522.

Howell-Adams, B., Wainwright, L. A. \& Seifert, H. S. (1996). The size and position of heterologous insertions in a silent locus differentially affect pilin recombination in Neisseria gonorrhoeae. Mol Microbiol 22, 509-522.

Kellogg, D. S., Jr, Peacock, W. L., Deacon, W. E., Brown, L. \& Pirkle, C. I. (1963). Neisseria gonorrhoeae. I. Virulence genetically linked to colonial variation. J Bacteriol 85, 1274-1279.

Kenyon, C. J. \& Walker, G. C. (1980). DNA-damaging agents stimulate gene expression at specific loci in Escherichia coli. Proc Natl Acad Sci U S A 77, 2819-2823.

Koomey, J. M. \& Falkow, S. (1987). Cloning of the $r e c A$ gene of Neisseria gonorrhoeae and construction of gonococcal recA mutants. J Bacteriol 169, 790-795.

Koomey, M., Gotschlich, E. C., Robbins, K., Bergstrom, S. \& Swanson, J. (1987). Effects of recA mutations on pilus antigenic variation and phase transitions in Neisseria gonorrhoeae. Genetics 117, 391-398.

Kowalczykowski, S. C., Dixon, D. A., Eggleston, A. K., Lauder, S. D. \& Rehrauer, W. M. (1994). Biochemistry of homologous recombination in Escherichia coli. Microbiol Rev 94, 401-465. 
Kushner, S. R., Nagaishi, H., Templin, A. \& Clark, A. J. (1971). Genetic recombination in Escherichia coli: the role of exonuclease I. Proc Natl Acad Sci US A 68, 824-827.

Lloyd, R. G. \& Thomas, A. (1983). On the nature of the RecBC and RecF pathways of conjugal recombination in Escherichia coli. Mol Gen Genet 190, 156-161.

Long, C. D., Madraswala, R. N. \& Seifert, H. S. (1998). Comparisons between colony phase variation of Neisseria gonorrhoeae FA1090 and pilus, pilin, and S-pilin expression. Infect Immun 66, 1918-1927.

McDaniel, L. S., Rogers, L. H. \& Hill, W. E. (1978). Survival of recombination-deficient mutants of Escherichia coli during incubation with nalidixic acid. J Bacteriol 134, 1195-1198.

Mehr, I. J. \& Seifert, H. S. (1998). Differential roles of homologous recombination pathways in Neisseria gonorrhoeae pilin antigenic variation, DNA transformation, and DNA repair. Mol Microbiol 30, 697-710.

Mehr, I. J., Long, C. D., Serkin, C. D. \& Seifert, H. S. (2000). A homologue of the recombination-dependent growth gene, $r d g C$, is involved in gonococcal pilin antigenic variation. Genetics 152, 523-532.

Miller, J. H. (1992). A Short Course in Molecular Genetics. Cold Spring Harbor, NY: Cold Spring Harbor Laboratory.

Miller, R. V. \& Kokjohn, T. A. (1990). General microbiology of recA: environmental and evolutionary significance. Annu Rev Microbiol 44, 365-394.

Mount, D. W., Low, K. B. \& Edmiston, S. J. (1972). Dominant mutations (lex) in Escherichia coli $\mathrm{K}-12$ which affect radiation sensitivity and frequency of ultraviolet light-induced mutations. J Bacteriol 112, 886-893.

Papavinasasundaram, K. G., Movahedzadeh, F., Keer, J. T., Stoker, N. G., Colston, M. J. \& Davis, E. O. (1997). Mycobacterial recA is cotranscribed with a potential regulatory gene called recX. Mol Microbiol 24, 141-153.

Papavinasasundaram, K. G., Colston, M. J. \& Davis, E. O. (1998). Construction and complementation of a recA deletion mutant of Mycobacterium smegmatis reveals that the intein in $\mathrm{Myco-}$ bacerium tuberculosis recA does not affect RecA function. Mol Microbiol 30, 525-534.

Roe, B. A., Clifton, S. \& Dyer, D. W. (1997). Gonococcal Genome Sequencing Project. http://www.genome.ou.edu/gono.html.

Sambrook, J., Fritsch, E. F. \& Maniatis, T. (1989). Molecular Cloning: a Laboratory Manual. Cold Spring Harbor, NY : Cold Spring Harbor Laboratory Press.

Sano, Y. (1993). Role of the recA-related gene adjacent to the recA gene in Pseudomonas aeruginosa. J Bacteriol 175, 2451-2454.

Seifert, H. S. (1997). Insertionally inactivated and inducible recA alleles for use in Neisseria. Gene 188, 215-220.

Seifert, H. S., Ajioka, R. S., Paruchuri, D., Heffron, F. \& So, M. (1990). Shuttle mutagenesis of Neisseria gonorrhoeae: pilin null mutations lower DNA transformation competence. J Bacteriol $172,40-46$.

Seifert, H. S., Wright, C. J., Jerse, A. E., Cohen, M. S. \& Cannon, J. G. (1994). Multiple gonococcal pilin antigenic variants are produced during experimental human infections. J Clin Invest $\mathbf{9 3 ,}$ 2744-2749.

Skaar, E. P., Lazio, M. P. \& Seifert, H. S. (2002). Roles of the recJ and $r e c N$ genes in homologous recombination and DNA repair pathways of Neisseria gonorrhoeae. J Bacteriol 184, 919-927.

Sparling, P. F. (1966). Genetic transformation of Neisseria gonorrhoeae to streptomycin resistance. J Bacteriol 92, 1364-1371.

Stohl, E. A. \& Seifert, H. S. (2001). The recX gene potentiates homologous recombination in Neisseria gonorrhoeae. Mol Microbiol 40, 1301-1310.

Story, R. M., Weber, I. T. \& Steitz, T. A. (1992). The structure of the E. coli recA protein monomer and polymer. Nature 355, 318-325.

Swanson, J., Kraus, S. J. \& Gotschlich, E. C. (1971). Studies on gonococcus infection. I. Pili and zones of adhesion: their relation to gonococcal growth patterns. J Exp Med 134, 886-906.

Swanson, J., Bergstrom, S., Robbins, K., Barrera, O., Corwin, D. \& Koomey, J. M. (1986). Gene conversion involving the pilin structural gene correlates with pilus ${ }^{+}$in equilibrium with pilus ${ }^{-}$ changes in Neisseria gonorrhoeae. Cell 47, 267-276.

Swanson, J., Robbins, K., Barrera, O., Corwin, D., Boslego, J., Ciak, J., Blake, M. \& Koomey, J. M. (1987). Gonococcal pilin variants in experimental gonorrhea. J Exp Med 165, 1344-1357.

Vierling, S., Weber, T., Wohlleben, W. \& Muth, G. (2000). Transcriptional and mutational analyses of the Streptomyces lividans recX gene and its interference with RecA activity. J Bacteriol 182, 4005-4011.

Virji, M. \& Heckels, J. E. (1983). Antigenic cross-reactivity of Neisseria pili: investigations with type- and species-specific monoclonal antibodies. J Gen Microbiol 129, 2761-2768.

Walker, G. C. (1996). The SOS response of Escherichia coli. In Escherichia coli and Salmonella: Cellular and Molecular Biology, pp. 1400-1416. Edited by F. C. Neidhardt and others. Washington, DC: American Society for Microbiology.

Yang, M. K., Chou, M. E. \& Yang, Y. C. (2001). Molecular characterization and expression of the recX gene of Xanthomonas campestris pv. citri. Curr Microbiol 42, 257-263.

Zaitsev, E., Alexseyev, A., Lanzov, V., Satin, L. \& Clark, A. J. (1994). Nucleotide sequence between recA and alaSp in E. coli K12 and the sequence change in four recA mutations. Mutat Res 323, 173-177.

Received 7 December 2001; revised 24 January 2002; accepted 5 February 2002. 